\title{
The Ellipticity of Galaxy Clusters at a Large Radius
}

\section{Ruben Coronel ${ }^{1}$, Yuanyuan Zhang ${ }^{2}$}

${ }^{1}$ University of Michigan, ${ }^{2}$ Fermilab

\section{Background}

Galaxy clusters are the largest know gravitationally bound structures in the universe, consisting of hundreds to thousands of galaxies (Figure 1) Dark matter contributes the majorit of mass to galaxy clusters, and therefore has a strong influence on the arrangement of member galaxies. By determining the shape of galaxy clusters, we can figure out the shape of the dark matter, which is important for cosmology and galaxy formation studies.

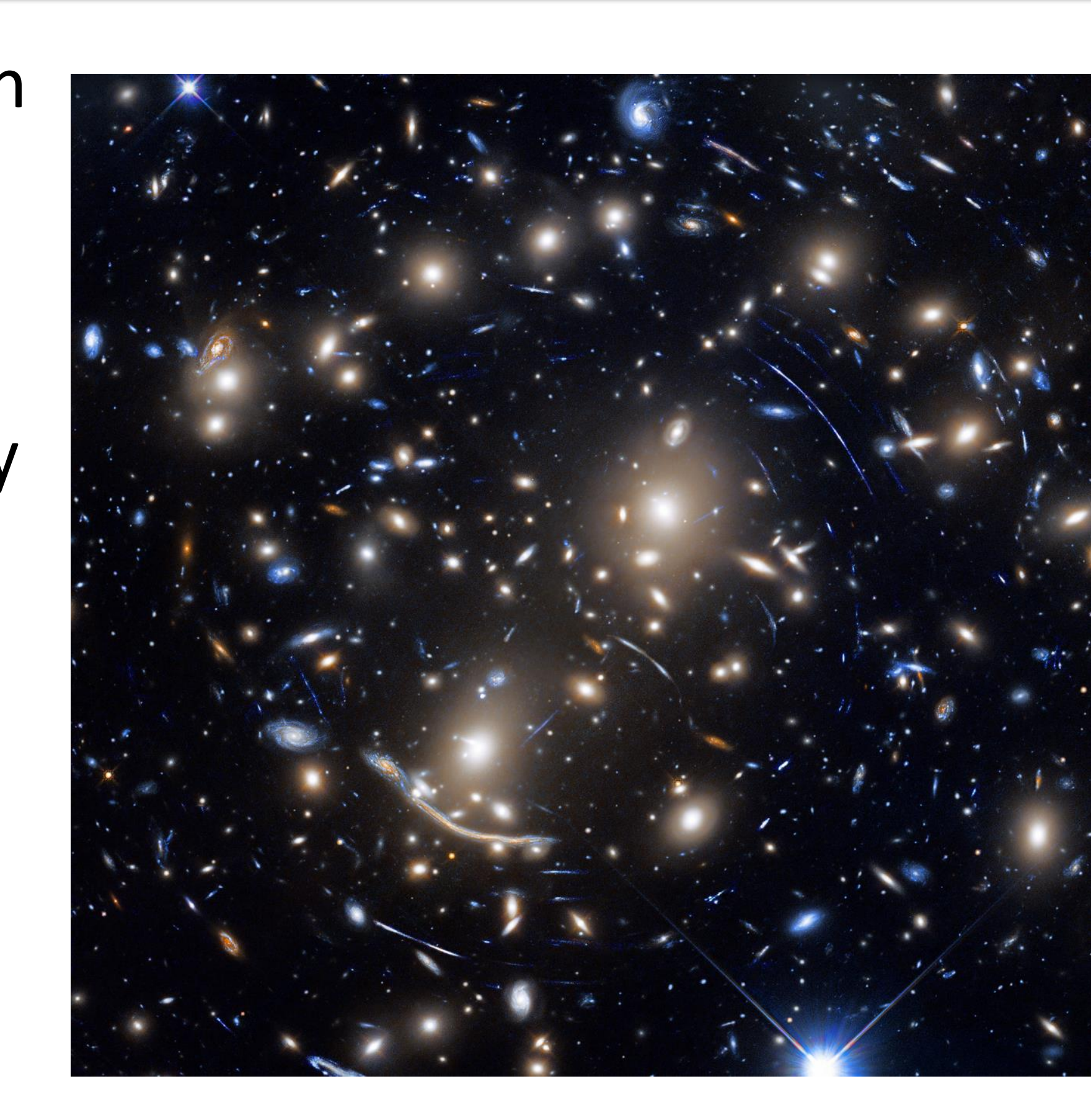

Figure 1: Galaxy cluster Abel 370 displaying gravitational lensing.

\section{Reproduction of Previous Work}

Cluster ellipticity, the degree to which the arrangement of satellite galaxies differs from being circular, has been previously measured to a small radius (Shin et al. 2018). Here we reproduce this work.
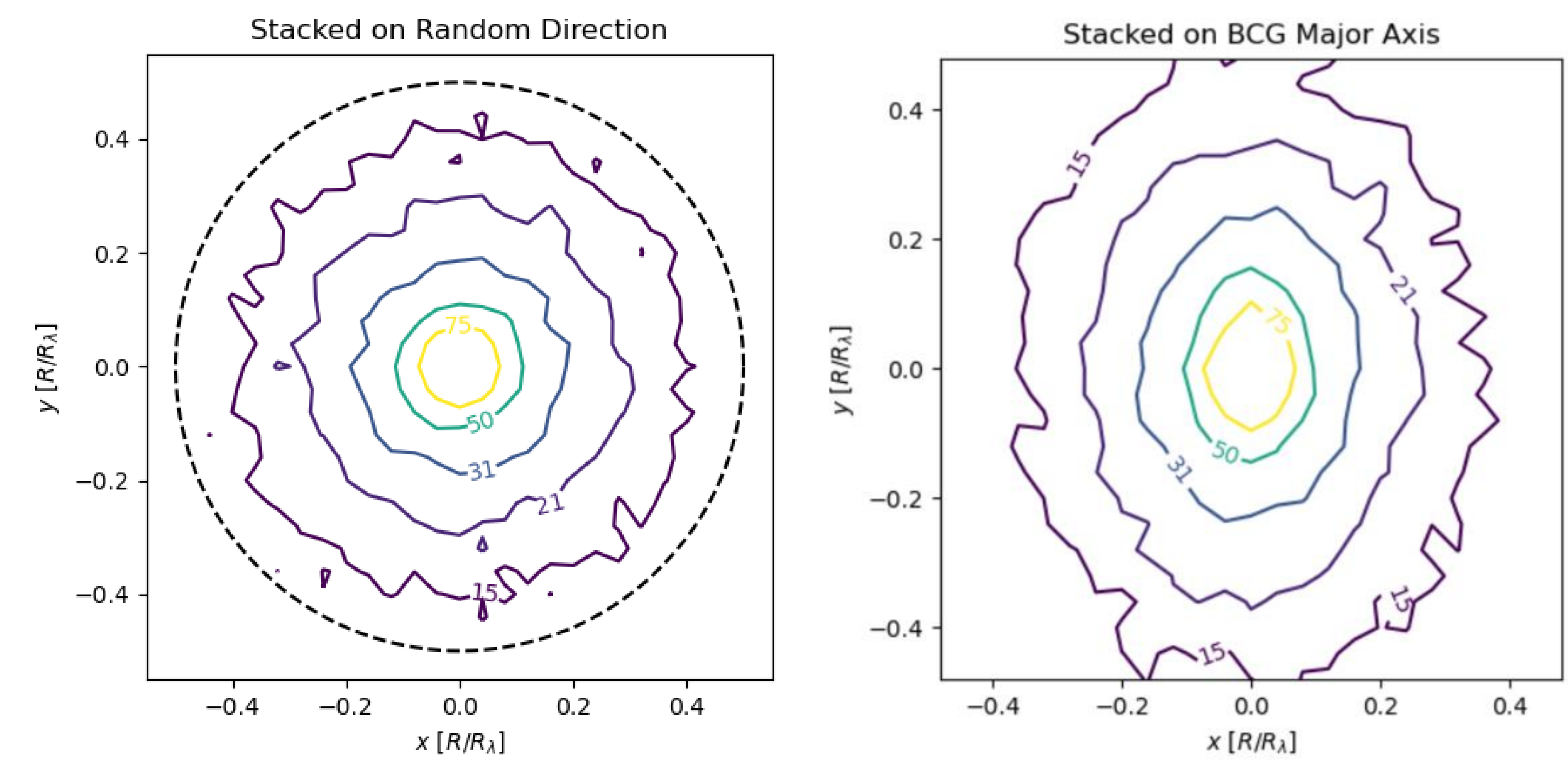

Figure 2 (left) and Figure 3 (right): Satellite density contours with random orientations (left) and stacked to align the major axes of the BCGs (right).

We use clusters in the DES redMaPPer catalog on Y1A1. Clusters are: - scaled by size $\mathrm{R}_{\lambda}$ (in $\mathrm{Mpc}$ )

- rotated such that the major axis of the BCG is vertical, and - "stacked" on top of each other.

We also stacked clusters without rotation to confirm that the ellipticity is purely caused by BCG alignment (Figure 2). The ellipticity of the contour lines in Figure 3 shows clear alignment between the major axis of the BCG and cluster satellites to a radius of approximately $0.5 R_{\lambda}$.

\section{Analysis of DES Y6A1 Data}

Our primary goal is to extend the measurement of cluster ellipticity to a much larger radius. We:

- Use redMaPPer clusters on the largest available DES dataset Y6A1 and select all galaxies within $10 \mathrm{Mpc}$ of each cluster center.

- Subtract a constant to account for unrelated background galaxies. - Scale, rotate, and stack clusters as described in the previous section.

In order to produce the background constant, we sample 1,000 random coordinates on the sky and cut to a radius of $10 \mathrm{Mpc}$

around each center, assuming some uniform redshift. The stacking procedure is applied to produce a 2D galaxy density histogram (Figure 4) for spatial bins surrounding the selected points.

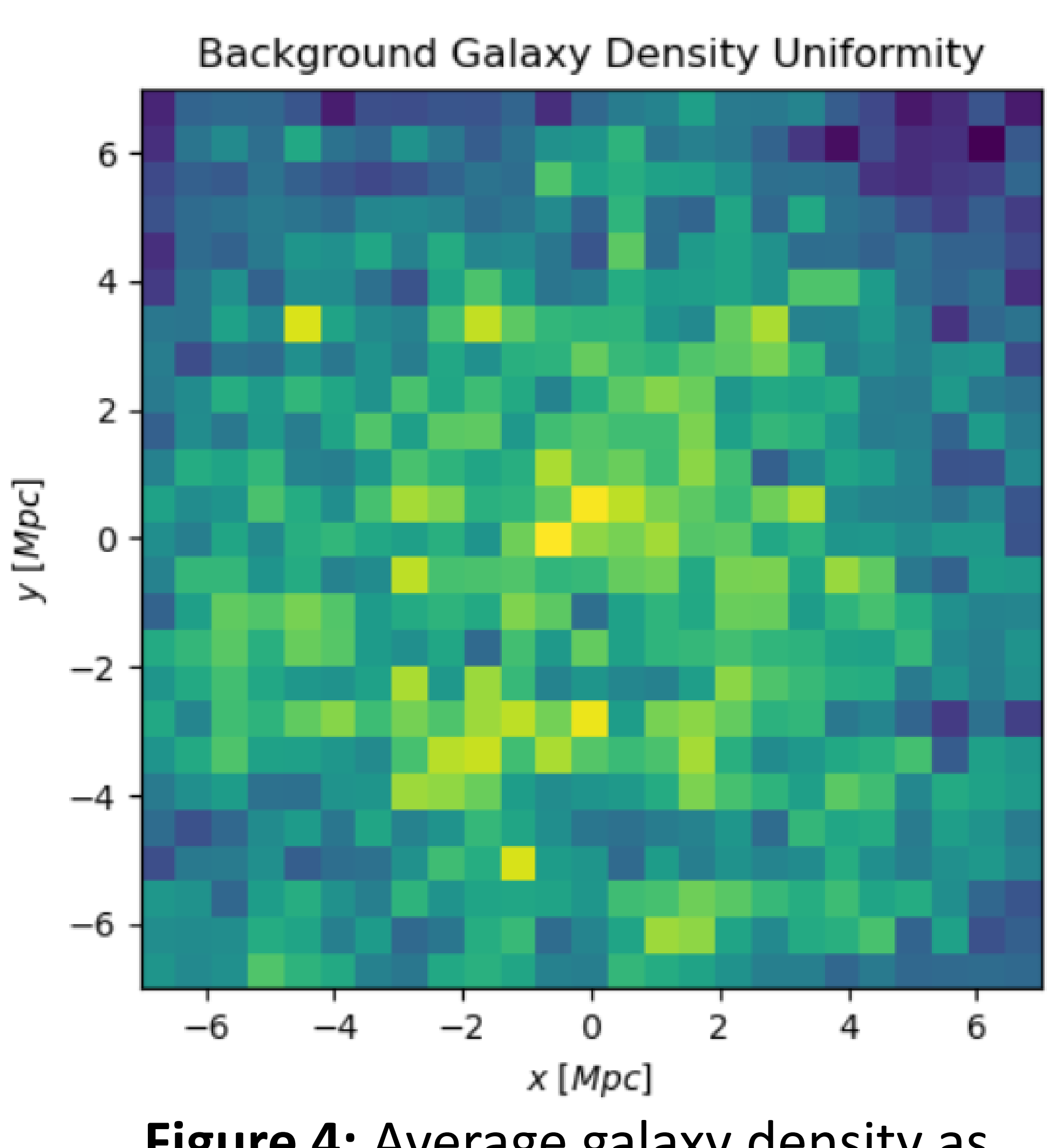

Figure 4: Average galaxy density as a function of spatial position.

Since the histogram is relatively symmetric and the density distribution is approximately normal both within and between clusters (Figures 7 and 8), we feel comfortable adopting a single value for the background constant. This value is adjusted according to the redshift and size $R_{\lambda}$ of each individual cluster.
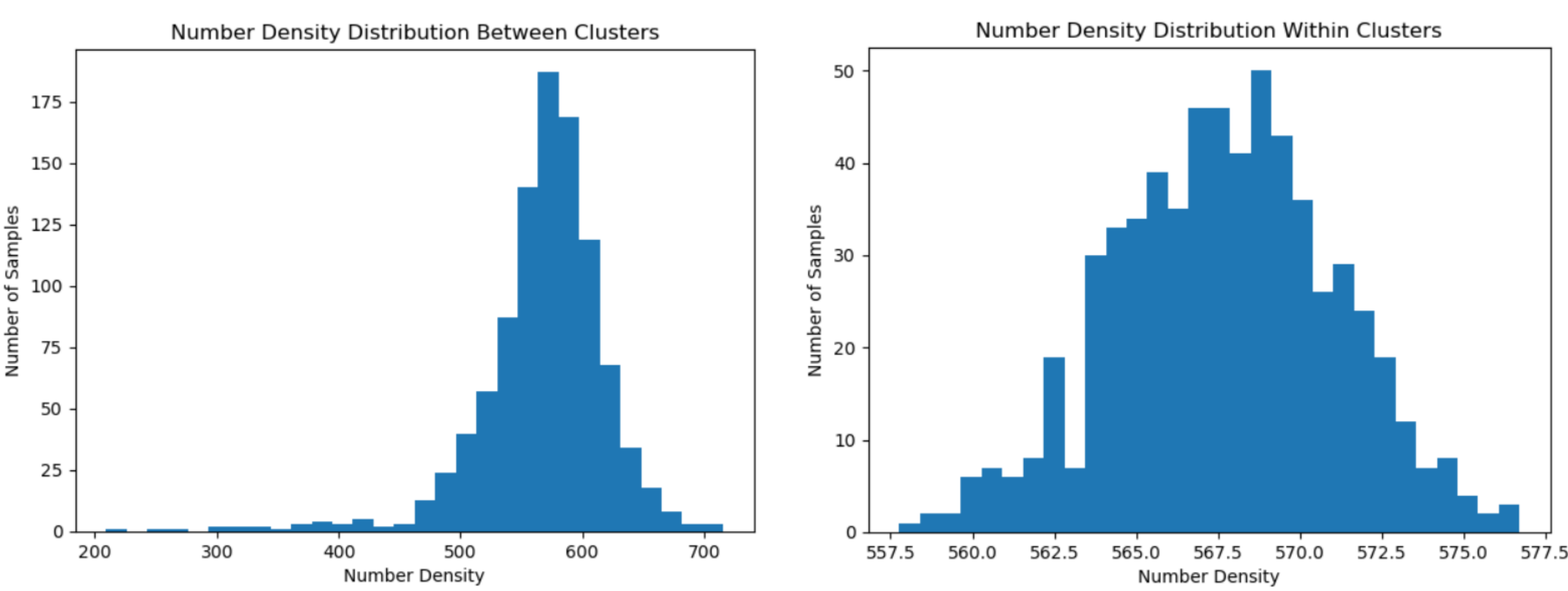

Figures 5 (left) and Figure 6 (right): Number density distribution within clusters, average density (averaged across all bins) between clusters.

\section{References}

Kiessling A., et al., 2015, Space Science Reviews, 193, 67 Shin T., et al., 2018, MNRAS, 475, 2421

\section{Results}

We find evidence of ellipticity extending to a radius well beyond previous measurements (Figure 7).

This observation is quantified in Figure 8, which shows a difference in the average number density on the vertical and horizontal axes of the contour plot that extends to a radius of approximately $5 \mathrm{R}_{\lambda}$, ten times larger than before.

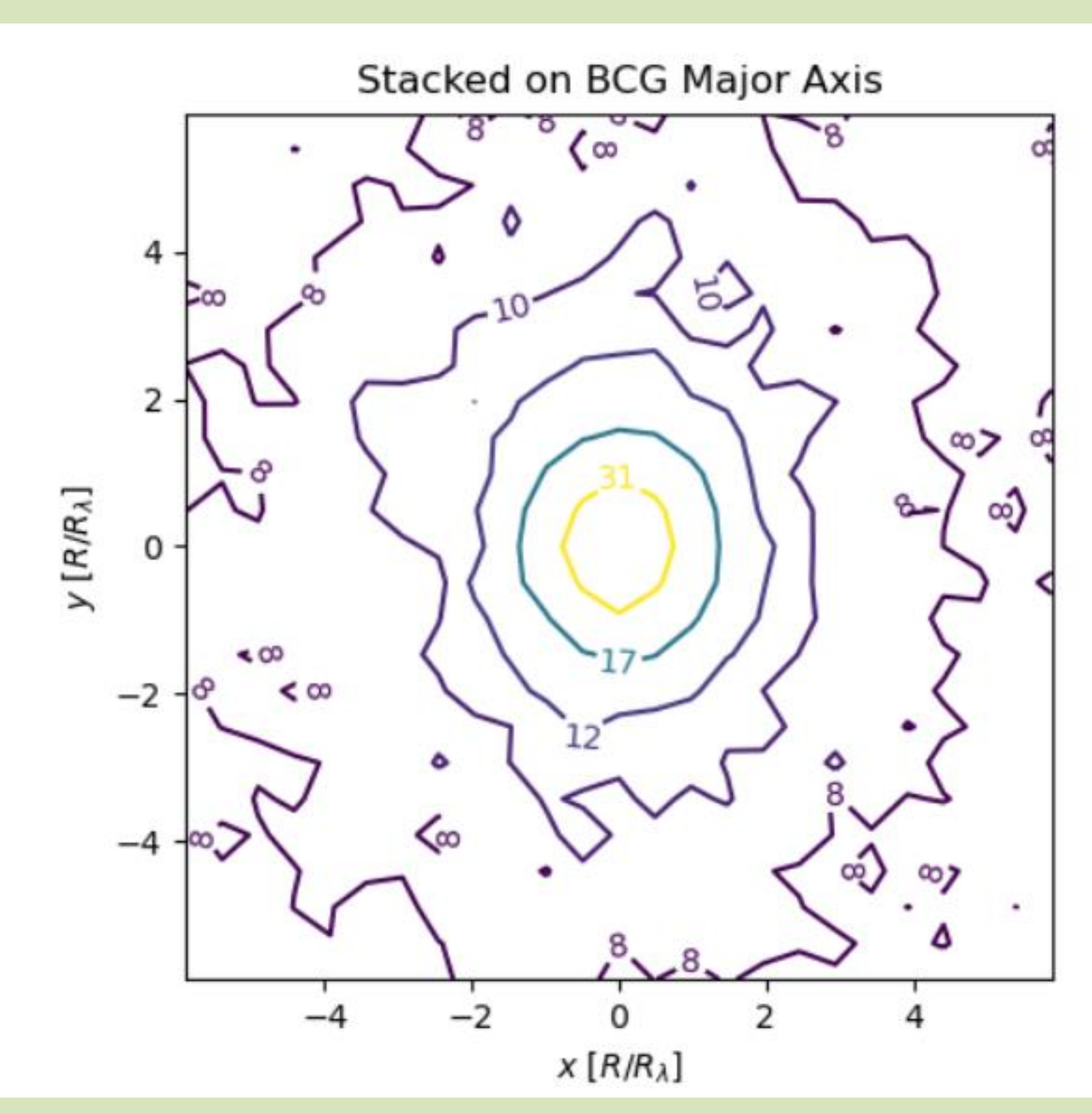

Figure 7 (top) and Figure 8 (bottom): Normalized satellite density contour and corresponding number density as a function of radius. Number density was calculated by taking the average of bins with equal radius on both the with and error bars were calculated using jackknife sampling.

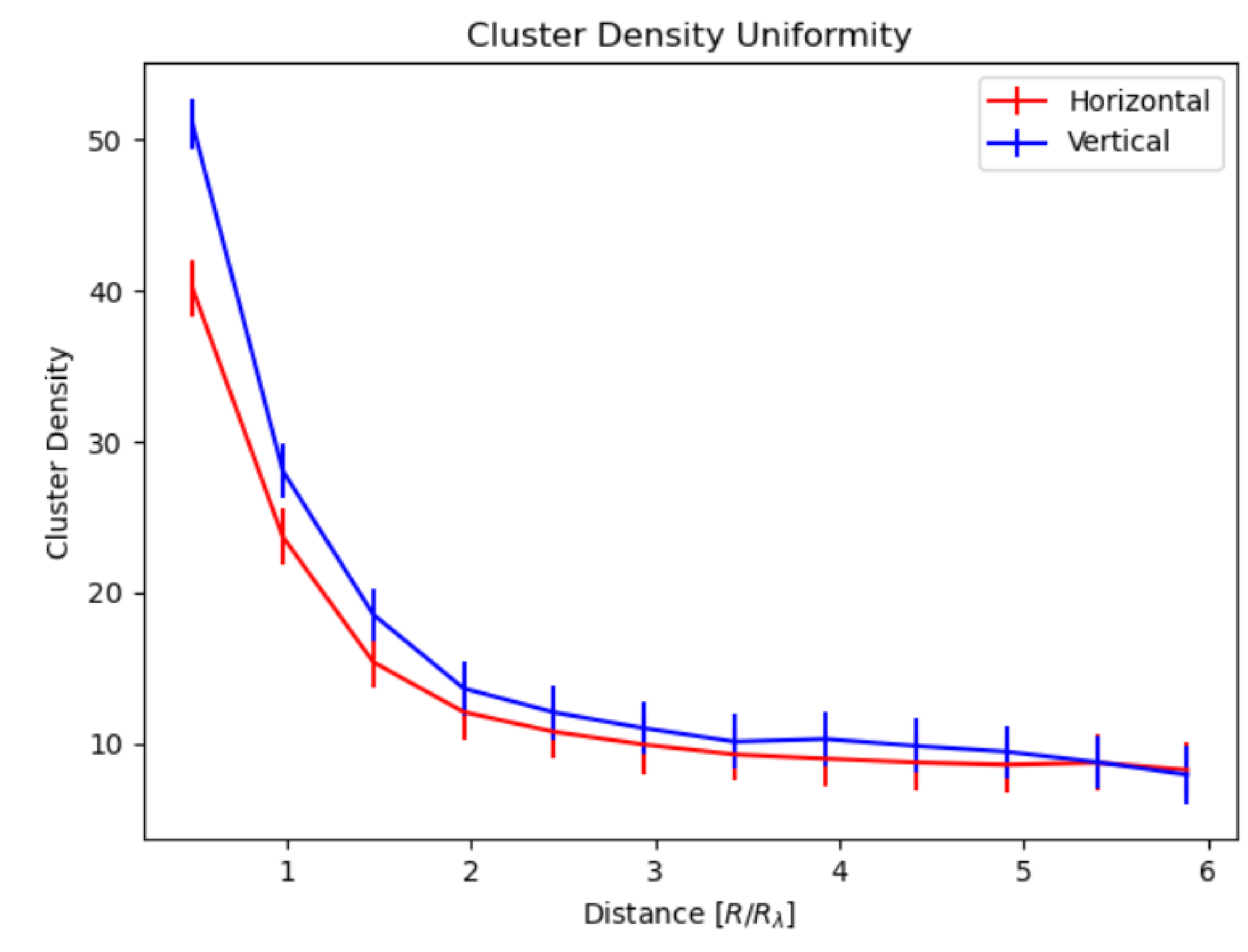

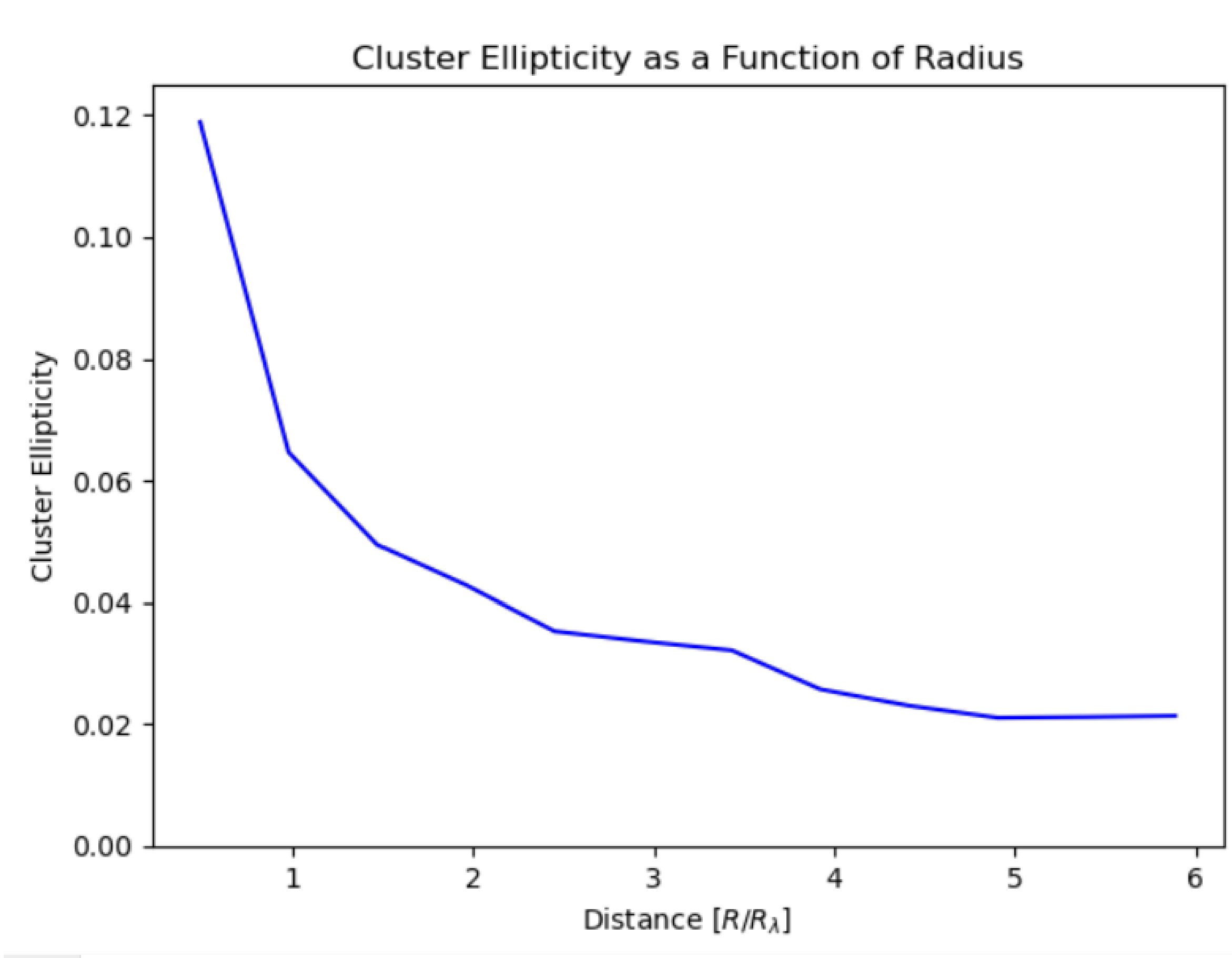

Figure 9: Cluster ellipticity decreases but remains present at comparatively large radii.
We further quantify the cluster ellipticity in 12 circular radial ranges as shown in Figure 9. As expected, the magnitude of ellipticity is largest near the center of the cluster, where satellite galaxies are more closely aligned with the BCG. However, a small ellipticity is still detected to much larger radii, confirming our previous findings. The significance of this result may be improved in future work by implementing redshift range limits during the satellite cutting process. 